\title{
Langages
}

http://www.necplus.eu/LGA

Additional services for Langages:

Email alerts: $\underline{\text { Click here }}$

Subscriptions: $\underline{\text { Click here }}$

Commercial reprints: $\underline{\text { Click here }}$

Terms of use : $\underline{\text { Click here }}$

\section{Odeurs et dimension hédonique à travers le prisme des adjectives}

Hélène Vassiliadou et Marie Lammert

Langages / Volume 2011 / Issue 181 / March 2011, pp 73 - 88

DOI: 10.3917/lang.181.0073, Published online: 03 April 2013

Link to this article: http://www.necplus.eu/abstract S0458726X11181053

How to cite this article:

Hélène Vassiliadou et Marie Lammert (2011). Odeurs et dimension hédonique à travers le prisme des adjectives. Langages, 2011, pp 73-88 doi:10.3917/lang.181.0073

Request Permissions : $\underline{\text { Click here }}$ 


\section{Odeurs et dimension hédonique à travers le prisme des adjectifs}

\section{INTRODUCTION ${ }^{1}$}

Il est généralement admis que l'on ne parvient pas à donner une description stable des odeurs parce qu'elles n'ont pas de parties que l'on puisse décrire. Elles ne présentent pas, en effet, de propriétés " objectives » que l'on pourrait saisir directement (cf. Rouby \& Sicard 1997). Pour autant, il existe des moyens linguistiques permettant de percer la carapace sémantique des odeurs, tels que différents descripteurs ${ }^{2}$ accompagnant le substantif odeur. Notre étude se limitera cependant aux adjectifs, postposés et antéposés, qui qualifient ce nom, car nous avons écarté d'autres types de descripteurs linguistiques tels que les compléments du nom (1) ou les relatives (2) :

(1) On ne distinguait plus la voûte de ce vaste carbet où s'accumulaient des odeurs de roucou, d'encens et de fumée. (Perret, Roucou, 1936)

(2) Une odeur qui tenait à la fois de celle des roses et des calices de l'oranger, mais fugitive et sauvage, achevait de donner je ne sais quoi de céleste à cette fleur mystérieuse que Séraphîtüs contemplait avec mélancolie [...]. (de Balzac, Séraphîta, 1846)

Ce choix, assez restrictif de prime abord, présente un avantage certain : les adjectifs étant non autonomes, ils permettent la formation de combinaisons très étroites avec le nom qu'ils accompagnent et constituent en même temps une

\footnotetext{
1. Un grand merci à G. Kleiber et à F. Gerhard-Krait pour nos discussions toujours aussi stimulantes.

2. Boisson (1997 : 38) exprimait déjà le besoin d'établir une liste de l'ensemble des descripteurs du nom odeur pour dégager les traits stables de l'objet examiné. Pour une présentation détaillée des descripteurs sensoriels, nous renvoyons aussi à Chauveix \& Egoroff (2003: 80-83).
} 
classe riche et assez homogène. L'adjectif joue alors le rôle qui lui est généralement reconnu, à savoir l'attribution de qualités (cf. Wierzbicka (1988:472) et Goes (1993 : 12), entre autres). Notre étude des adjectifs associés au substantif odeur peut ainsi être un moyen de décrire les propriétés de ce mot et, par là, un moyen indirect d'identifier les odeurs et de s'interroger sur l'existence de types d'odeurs.

Les travaux d'inspiration linguistique portant sur le domaine olfactif visent essentiellement à relier structures linguistiques et catégories cognitives ou bien encore approche linguistique et approche anthropologique ou sociologique. Leur protocole expérimental s'appuie sur des données provenant de la mise en discours d'expériences olfactives de sujets. Il s'agit d'une procédure qui permet aux sujets de communiquer leurs perceptions sensorielles en représentant l'objet odeur par des mots. Nous nous démarquons des études psycholinguistiques en ce que nous ne nous fondons pas sur une expérience qui vise à faire décrire les odeurs par les réponses à des questions du type "selon vous, qu'est-ce qu'une odeur? » ou « quels types d'odeurs distinguez-vous dans votre environnement $?{ }^{3}$. Nous partons de productions (écrites) attestées et non sollicitées, et l'originalité de notre étude se trouve dans la démarche suivie : classer et expliquer les combinaisons possibles du mot odeur avec des adjectifs permet de confirmer et/ou d'infirmer les résultats obtenus dans les études antérieures, mais aussi d'apporter de nouvelles données dans la description des odeurs ainsi que des éléments de réponse à la question de leur catégorisation, stable ou non (cf. aussi David \& Rouby 2002).

\section{PROTOCOLE D'ÉTUDE}

Nous avons d'abord établi un corpus, puis nous avons hiérarchisé, décrit et tenté d'expliquer les résultats obtenus. Nous avons ainsi effectué deux requêtes dans Frantext (version catégorisée) permettant de mettre au jour les adjectifs antéposés et postposés à odeur(s) ${ }^{4}$. Les données obtenues ont ensuite été traitées automatiquement à l'aide du concordancier Oxford Wordsmith. Nous avons enfin " nettoyé » ces résultats en écartant des adjectifs qui « servent à délimiter un

\footnotetext{
3. Cf. aussi le recours à des entretiens semi-directifs, à des questionnaires ouverts, à des dispositifs recréant une situation olfactive, etc. Les chercheurs étudient ainsi comment les personnes classent les odeurs en catégories. Une autre manière de procéder consiste à faire respirer à différents sujets un nombre X d'odeurs, puis de leur demander de faire des groupes suivant la similitude des odeurs (catégorisation dite libre). Cf. Chollet, Valentin \& Abdi (2003) ; Chréa et al. (2005) ; Chréa \& Valentin (2007). Même l'article de Cance et al. (2009), qui traite du lien entre forme adjectivale et sens de la vision et de l'audition, suit la même démarche méthodologique que les autres travaux en linguistique cognitive. Signalons toutefois le récent article de Dubois \& Resche-Rigon (2009) qui s'appuie sur un corpus de romans extrait de la base textuelle Frantext.

4. Nous avons établi nos requêtes de manière à ne faire apparaître, autant que faire se peut, qu'un seul adjectif associé à odeur(s). Cela donne : \&e(g!=A APr APs Cc) \&e(g=A APr Aps) \&modeur \&e(g!=A APr APs) pour les antéposés et \&e(g!=A APr APs) \&modeur \&e(g=A APr APs) \&e(g!=A APr APs Cc) \&e(g!=A APr APs) pour les postposés.
} 
objet de discours à l'intérieur de sa catégorie, sans le décrire » comme autre, même, telle, première, dernière, diverses, différentes, propre (au sens 'sienne') ou analogue, et qui ne nous renseignent pas directement sur les propriétés des odeurs (Noailly, 1999 : 102). Ces différentes manipulations ont abouti à un corpus A de 1150 occurrences d'adjectifs antéposés et à un corpus B de 1433 occurrences $\mathrm{d}^{\prime}$ adjectifs postposés ${ }^{5}$.

Cette démarche présente l'avantage de traiter des énoncés attestés, authentiques, portant sur différentes expériences olfactives, que ce soit dans un contexte mémoriel ou en lien direct avec une situation donnée. Elle allie ainsi une multiplicité de contextes à une multiplicité de points de vue. Elle permet également de faire ressortir les récurrences par le biais de la routine littéraire associée à telle ou telle odeur, routine qui n'est d'ailleurs pas absente dans le cas de sujets interrogés qui peuvent avoir les mêmes réflexes associatifs. Cette récurrence donne, par ailleurs, une vision de la manière dont sont habituellement décrites les odeurs. De ce fait, nous pouvons quand même parler d'une stabilité du lexique, d'un partage dans la description du ressenti olfactif. Nous pensons en somme que, l'hypothèse selon laquelle il existerait une culture olfactive propre à chacun d'entre nous est à relativiser.

Toutefois, cette méthode présente aussi des inconvénients. Le corpus pris en compte est un corpus littéraire : la spontanéité des énoncés est ainsi relativement limitée et les expériences olfactives relatées sont souvent issues de situations de fiction. Les descripteurs utilisés ne sont pas des descripteurs « directs », comme ils peuvent l'être dans les expériences menées ailleurs où l'on fait sentir des odeurs. Il s'agit plutôt d'éléments qui permettent de relever les récurrences dans la description des odeurs lorsque les locuteurs expriment le besoin ou l'envie de décrire ce qu'ils (res)sentent. L'étude des adjectifs associés à odeur dans Frantext constitue alors une première étape qui pourra être complétée par des analyses similaires dans d'autres types de corpus (journalistiques et oraux).

Pour notre partie descriptive, nous avons procédé dans un premier temps de manière « aveugle » dans l'établissement de nos classifications, c'est-à-dire que nous avons pris en compte les occurrences considérées en dehors de tout contexte. Puis, nous avons dû affiner ces résultats en recourant au contexte, aux définitions lexicographiques et à l'avis de plusieurs sujets parlants ; la difficulté majeure étant de hiérarchiser les informations sémantiques véhiculées par les adjectifs selon leurs traits principaux et secondaires (hédonique positif ou négatif, effet, propriété, source, temporalité, intensité, identification). De plus, le trait propriété recouvre plusieurs sous-traits selon qu'il s'agit d'une propriété olfactive pure (odoriférante), d'une propriété multisensorielle (suave) ou encore de la transposition de propriétés issues de différents domaines (dimensionnel,

5. Ces données seront traitées en prenant en compte deux facteurs : le nombre d'occurrences (tokens) des adjectifs utilisés dans le corpus (2 583 au total) ainsi que la diversité lexicale (types) à travers le nombre d'adjectifs différents employés (629). 
gustatif, tactile, etc., tels que petite, sucrée et molle). Aussi a-t-il fallu s'entendre à la fois sur la pertinence des traits retenus et sur leur ordre d'apparition. Et malgré tout le soin que nous y avons apporté, le classement proposé, nous le reconnaissons fort volontiers, peut encore donner lieu à discussion. Quoi qu'il en soit, une première incursion dans le corpus permet de constater que la prégnance de la dimension hédonique généralement avancée concernant les odeurs est largement confirmée et qu'elle se manifeste de différentes manières. En témoigne, tout d'abord, le nombre d'adjectifs axiologiques parmi les adjectifs les plus fréquemment utilisés avec odeur(s), comme l'illustrent les tableaux suivants :

\begin{tabular}{|c|c|c|c|}
\hline Adj. + odeur & Nbre d'occ. & Adj. + odeur & Nbre d'occ. \\
\hline bonne(s) odeur(s) ${ }^{6}$ & 255 & écœurante(s) odeur(s) & 14 \\
\hline forte(s) odeur(s) & 104 & violente odeur & 14 \\
\hline mauvaise(s) odeur(s) & 79 & chaude(s) odeur(s) & 13 \\
\hline âcre(s) odeur(s) & 46 & délicieuse(s) odeur(s) & 12 \\
\hline vague(s) odeur(s) & 43 & affreuse odeur & 11 \\
\hline douce(s) odeur(s) & 27 & faible odeur & 11 \\
\hline légère(s) odeur(s) & 23 & pénétrante(s) odeur(s) & 11 \\
\hline suave(s) odeur(s) & 22 & aigre odeur & 10 \\
\hline fade(s) odeur(s) & 19 & fraîche odeur & 10 \\
\hline puissante(s) odeur(s) & 18 & insupportable odeur & 10 \\
\hline
\end{tabular}

\section{Tableau 1 : Corpus A - Adjectifs les plus fréquents (adjectifs hédoniques grisés)}

\begin{tabular}{|l|c|l|c|}
\hline Odeur + Adj. & Nbre d'occ. & Odeur + Adj. & Nbre d'occ. \\
\hline odeur(s) forte(s) & 83 & odeur(s) douce(s) & 16 \\
\hline odeur(s) fade(s) & 78 & odeur(s) particulière & 16 \\
\hline odeur(s) âcre(s) & 37 & odeur(s) sucrée(s) & 16 \\
\hline odeur(s) fétide(s) & 30 & odeur(s) mêlée(s) & 15 \\
\hline odeur(s) infecte(s) & 29 & odeur(s) indéfinissable(s) & 14 \\
\hline odeur(s) nauséabonde(s) & 28 & odeur(s) fauve(s) & 13 \\
\hline odeur(s) chaude(s) & 23 & odeur amère & 12 \\
\hline odeur(s) délicieuse(s) & 23 & odeur enivrante(s) & 12 \\
\hline odeur pénétrante & 21 & odeur(s) lourde(s) & 12 \\
\hline odeur(s) désagréable(s) & 19 & odeur douceâtre & 11 \\
\hline odeur(s) écœurante(s) & 17 & odeur(s) violente(s) & 11 \\
\hline odeur(s) fraîche(s) & 17 & odeur(s) marine(s) & 10 \\
\hline odeur(s) puissante(s) & 17 & odeur(s) musquée(s) & 10 \\
\hline odeur(s) suave(s) & 17 & odeur(s) sauvage(s) & 10 \\
\hline odeur aigre & 16 & odeur(s) végétale(s) & 10 \\
\hline
\end{tabular}

Tableau 2 : Corpus B - Adjectifs les plus fréquents (adjectifs hédoniques grisés)

L'étiquette hédonique regroupe les adjectifs permettant d'exprimer un jugement qualitatif sur l'odeur, selon deux grands axes, constitués par les bonnes et les mauvaises odeurs. Nous incluons dans ces adjectifs à la fois des adjectifs purement hédoniques (du type bonne, mauvaise) qui véhiculent lexicalement une évaluation positive ou négative et d'autres, à l'extension plus restreinte, qui peuvent avoir d'abord un trait de propriété (âcre, aigre), d'effet (nauséabonde, irritante) ou de source (excrémentielle, cadavéreuse) auquel est associé, à un degré second, un aspect hédonique. Ces deux types d'adjectifs décrivent l'effet - agréable ou désagréable -

6. Les adjectifs bonne, mauvaise, agréable et désagréable constituent le groupe de tête dans le profil combinatoire dressé par Blumenthal (2011). 
que l'odeur produit sur celui qui la sent. Ils se distinguent cependant par le fait que les seconds apportent une information sur l'origine de l'impression agréable ou désagréable tandis que les premiers se limitent à une information hédonique. Nous avons enfin écarté les adjectifs tels que piquante qui dénotent certes un effet produit sur celui qui sent, mais sans inclure de jugement positif ou négatif sur l'odeur qualifiée de cette manière. Ainsi, on pourra aussi bien avoir une odeur piquante de poivre frais qu'une odeur piquante d'eau de javel.

Par ailleurs, les adjectifs hédoniques totalisent $55.05 \%$ des occurrences relevées dans les corpus $A$ et $B$. Ils sont donc très largement représentés. Ces données nous ont poussées à examiner plus avant la catégorie des adjectifs hédoniques, et plus particulièrement le lien entre la dimension hédonique et la position (antéposition/postposition) que les adjectifs correspondants occupent par rapport au nom. Nous avons ainsi étudié, à l'intérieur de cette classe, la proportion d'adjectifs positifs et négatifs afin de proposer quelques explications sur la prégnance de cet axe ${ }^{7}$. Notre analyse a abouti à un classement de ces adjectifs selon qu'ils sont des hédoniques purs ou qu'ils présentent un, voire deux traits supplémentaires (propriété, effet, intensité, source, etc.). L'examen d'adjectifs relevant exclusivement $\mathrm{d}^{\prime}$ autres catégories telles que la source (printanière, métallique) ou l'identification ${ }^{8}$ par exemple (vague, indéfinissable, indescriptible) fera l'objet d'un travail ultérieur. Signalons simplement que les adjectifs qui spécifient la source ne sont pas particulièrement privilégiés en position pré-nominale, mais qu'ils sont assez présents en postposition ${ }^{9}$. En outre, nous avons pu observer que les adjectifs liés à la temporalité (tels que persistante, tenace, continuelle, intermittente, perpétuelle, éternelle, furtive, sempiternelle, ineffaçable, indélébile, longue, lente) sont assez nombreux, contrairement à ce qui a été avancé dans d'autres études (cf. surtout David 2002 ; David \& Dubois en ligne a). Selon ces auteurs, l'odeur n'aurait pas de propriétés temporelles en français. Toutefois, il ne faut pas mettre sur le même plan unité temporelle et dimension temporelle : l'odeur n'est pas une unité temporelle telle que jour ou heure, mais elle est soumise à la dimension temporelle. Par conséquent, il est évident que l'on ne peut pas avoir de séquences du type après l'odeur ou pendant l'odeur comme c'est le cas de pendant le film qui a comme dimension principale le temps. Pour notre part, nous regroupons sous cette étiquette les adjectifs décrivant la durée de la sensation.

\footnotetext{
7. Boisson (1997 : 34) parle de l'importance « frappante de la variable évaluative, 'hédoniste' ». On constate, d'ailleurs, que la plupart des termes olfactifs sont connotés positivement ou négativement. Cf. aussi Buck (1949) ; Schleidt et al. (1988) ; David et al. (1997) ; Holley (1997) ; Ricaud (2004) ; Candau \& Jeanjean (2006); Dubois (2000, 2006).

8. Le terme identification désigne différents types d'adjectifs dont ceux signifiant qu'il est difficile d'associer une étiquette particulière à une odeur donnée.
}

9. Notons toutefois que le trait source est plus souvent exprimé par le biais d'une structure $<$ N1 de N2 $>$. 


\section{LA DIMENSION HÉDONIQUE EN RELATION AVEC LA POSITION DE L'ADJECTIF}

Il est tout d'abord intéressant de noter que les hédoniques dominent en antéposition : $67.30 \%$ des occurrences d'adjectifs du corpus A sont des hédoniques, contre $45.22 \%$ dans le corpus B. Ces données doivent toutefois être pondérées, car l'adjectif bonne, en antéposition, représente à lui seul un tiers des occurrences d'adjectifs hédoniques (et deux tiers des adjectifs hédoniques positifs). Cela n'a rien d'étonnant car, selon la littérature consacrée à la place de l'adjectif épithète en français, une forte tendance à l'antéposition caractérise les adjectifs de ce type, dits élémentaires ou à sens réduit, qui, en vertu de leur très grande extension, peuvent se combiner avec un nombre élevé de substantifs ${ }^{10}$. B. Larsson (1994: 39) note ainsi que les adjectifs de « valorisation positive » sont généralement antéposés. Outre le critère sémantique, les propriétés morphophonologiques des adjectifs constituent, comme on le sait, un critère décisif en la matière (cf. à ce propos les relevés de M. Wilmet (1981) dans lesquels les adjectifs les plus souvent antéposés sont monosyllabiques : grand, bon, beau, fort). Enfin, dans la perspective syntaxique en termes de " poids » d'A. Abeillé et D. Godard (1999), les adjectifs du type bon sont analysés comme éléments « légers » (formes syntaxiquement faibles) mettant en jeu une « combinaison directe ou quasi lexicale » avec le nom, tandis que la postposition implique une combinaison syntagmatique.

L'importance de l'axe hédonique en antéposition est aussi soulignée par la variété des adjectifs présentant ce trait : $35.44 \%$ des adjectifs employés en antéposition sont hédoniques alors qu'ils ne sont que $26 \%$ en postposition. Ces observations - qui rejoignent d'ailleurs la thèse selon laquelle l'antéposition correspond à une saisie globale, la postposition à une saisie analytique - corroborent l'idée que l'axe hédonique est " précompacté » et que les odeurs sont appréhendées en première instance comme bonnes ou mauvaises. De plus, ce fait syntaxique trouve un corollaire explicatif en termes d'iconicité : la conceptualisation de la propriété se fait préalablement à celle du support (cf. Honeste 2005). Des remarques avancées dans le domaine de la cognition confortent nos données : la très forte tendance des adjectifs hédoniques à l'antéposition suggère, en effet, que la dimension hédonique des odeurs est appréhendée avant et à l'exclusion de toute autre propriété. Le fait d'avoir parmi les premières places des collocations du type bonnes/mauvaises odeurs signifierait qu'il s'agit de catégories préétablies et préconçues, à l'opposé d'une mince odeur (deux occurrences dans notre corpus) qui n'est pas une caractérisation consensuelle. On notera d'ailleurs que, selon G. Le Bidois et R. Le Bidois (1967), l'antéposition signale une qualification banale, non-réfléchie (cf. Forsgren 1978). De même, H. Nølke (2000 : 521) rappelle que les adjectifs antéposés ont « un sens atténué et plus 
ou moins général », et que l'on observe souvent "une fusion sémantique et prosodique (la liaison est habituelle) avec le substantif ».

La variable positive ou négative des hédoniques nous apporte, quant à elle, d'autres informations sur l'appréhension des odeurs. Selon J.-M. Hombert (1992) et C. Boisson (1997), les aspects négatifs sont plus « remarquables » que les aspects positifs en ce sens que, nous vivons dans un monde plutôt neutre olfactivement et dans lequel les mauvaises odeurs sont, au fil du temps, devenues intolérables ${ }^{11}$. Il semble d'ailleurs exister une dissymétrie entre mauvaises et bonnes odeurs : les premières frappent plus notre sensibilité que les secondes, ce que confirme la fréquence relative des adjectifs hédoniques positifs et négatifs, ceux-ci étant plus fréquents que ceux-là. Notre investigation confirme que le pôle négatif est plus important que le pôle positif : antéposés ou postposés, les adjectifs hédoniques négatifs sont les plus utilisés, que ce soit en nombre d'occurrences (exemplaires/tokens) ou en nombre d'adjectifs différents (types). En antéposition, leur avance est cependant assez courte $(51.42 \%$ d'occurrences de négatifs contre $48.58 \%$ de positifs et 44 adjectifs hédoniques négatifs contre 29 adjectifs hédoniques positifs), mais elle est néanmoins remarquable compte tenu de la prédominance déjà soulignée de l'adjectif bonne. La postposition marque une opposition très nette entre hédoniques négatifs et positifs : $79.48 \%$ des hédoniques postposés sont négatifs et ils ne sont que $20.52 \%$ de positifs. Cette différence va de pair avec le nombre d'adjectifs utilisés : le corpus B contient 79 adjectifs hédoniques négatifs et 31 adjectifs hédoniques positifs. Ces observations vont dans le sens de C. Boisson (1997 : 38) pour qui « le lexique des mauvaises odeurs est plus fourni et plus diversifié que le lexique des bonnes odeurs, et cela d'une manière très significative ». Il est souvent $d$ 'ailleurs stipulé dans la littérature cognitive qu'un tel phénomène s'expliquerait par le fait que les gens ont généralement tendance à chercher à définir le mauvais (cf. en quoi ça sent mauvais ?). Toutefois, il reste possible de dire « ça pue ! » ou « ça sent ! » sans qu'il ne soit nécessaire de spécifier plus avant. Les adjectifs négatifs sont majoritairement postposés pour une raison connue : linguistiquement, le négatif se construit sur le positif, ce qui correspond à la thèse de la saisie analytique mentionnée supra.

Si on analyse de plus près la diversité lexicale des adjectifs hédoniques, on constate que la majorité de ceux qui relèvent du pôle positif donnent une information strictement hédonique. Près de la moitié des adjectifs hédoniques positifs postposés (14 sur 31) et un peu moins de la moitié des antéposés (12 sur 29) sont de purs hédoniques : ils ne contiennent pas d'autres traits lexicaux. En termes d'occurrences d'adjectifs, cette particularité est encore plus marquée pour les antéposés, étant donné la prégnance de l'adjectif bonne. $76.86 \%$ des occurrences d'adjectifs hédoniques antéposés positifs sont ainsi de purs hédoniques et c'est le cas de $43.61 \%$ des occurrences d'adjectifs hédoniques positifs postposés. Les

11. Cf. Corbin (1982) qui décrit « l'entreprise de désodorisation » conduite en Occident à partir de 1750 Cf. aussi Candau \& Jeanjean (2006). 
hédoniques négatifs purs, en revanche, constituent - pour ce qui est du nombre d'adjectifs dédiés à cette catégorie - un tiers des antéposés (14 sur 44) et moins d'un quart des postposés (18 sur 79). La prise en compte du nombre d'occurrences dans le corpus accentue encore davantage cet écart auprès des postposés, puisque $1 / 10^{\mathrm{e}}$ des occurrences d'adjectifs hédoniques négatifs postposés sont de purs hédoniques, les antéposés de ce type occupant les mêmes proportions que pour la diversité lexicale, c'est-à-dire $1 / 3$ des occurrences. Beaucoup d'adjectifs qui relèvent du pôle hédonique négatif expriment des traits supplémentaires. $\mathrm{D}^{\prime}$ autres dimensions entrent ainsi en compte dans la description des odeurs jugées comme négatives ou positives, que ce soit en antéposition ou en postposition. Ces dimensions nous permettront également d'esquisser $\mathrm{d}^{\prime}$ une certaine manière le prototype d'une bonne ou d'une mauvaise odeur.

\section{LA DIMENSION HÉDONIQUE ASSOCIÉE À D'AUTRES TRAITS}

Afin de discriminer les adjectifs qui associent à l'indication hédonique un trait supplémentaire, nous pouvons utiliser le test suivant :

Si je la trouve ignoble, c'est parce qu'elle est suffocante

vs

*Si je la trouve suffocante, c'est parce qu'elle est ignoble.

L'adjectif ignoble ne nous dit rien sur l'odeur elle-même; quant à suffocante, hors contexte, il n'indique que l'effet produit par l'odeur, sans spécifier d'où vient cette propriété. Quand un adjectif associe au trait hédonique une autre information, il s'agit majoritairement - dans notre corpus - des traits propriété, intensité et effet. Ainsi, pour qu'une odeur puisse faire suffoquer quelqu'un, elle doit posséder des propriétés induisant une sensation d'étouffement. Pour ce qui est des autres dimensions, l'identification est quasi inexistante et ne concerne que les hédoniques positifs antéposés avec deux adjectifs, extraordinaire et chère :

(3) Une extraordinaire odeur d'eau de Cologne et d'incendie emplit le bureau aux vitres brisées : une parfumerie brûlait. (Malraux, L'Espoir, 1937)

(4) [...] pure émanation divine qui mêlait en moi ta douceur à la petite senteur fine des longues tresses d'une sœur, chère odeur, tu t'en es allée [...] (Sully Prudhomme, Les Vaines tendresses, 1875)

Extraordinaire appartient à la classe des adjectifs qui délimitent une sous-classe de $\mathrm{N}$ identifiables par leur rareté (comme c'est aussi le cas d'exceptionnel, cf. Larsson 1994) et chère indique que le locuteur reconnaît, donc identifie, une odeur déjà connue. Quant à la source, elle est plus présente en postposition qu'en antéposition. Toutefois, il y a très peu d'adjectifs combinant hédonisme et source et ceux-ci sont alors négatifs (cadavérique, putride, urineuse, excrémentielle). 


\subsection{Les traits hédonique et propriété}

La catégorie propriété regroupe deux ensembles. Il y a, d'une part, les adjectifs liés principalement à la perception sensorielle et, plus particulièrement, ceux qui sont relatifs aux « dimensions de la matière ». La matière est, en effet, ouverte à tous les sens puisque l'on peut la goûter (amère), la toucher (rugueuse), la sentir (odoriférante), la mesurer (lourde, longue, épaisse), la décrire (limpide, onctueuse, vilaine) ; ces différentes activités faisant intervenir une ou plusieurs appréhensions sensorielles simultanées. D'autre part, il y a les adjectifs de propriétés physiques du vecteur de l'odeur (i.e. l'air), ces propriétés étant sensoriellement perceptibles telles que les sensations thermiques (chaude, fraîche, tiède) (cf. Boisson 1997).

Les adjectifs qui combinent le trait " propriété » avec la caractéristique hédonique indiquent que le caractère agréable ou désagréable de l'odeur provient de la propriété qu'elle possède. Ainsi, les adjectifs douce et suave se distinguent des hédoniques purs bonne et délicieuse. De même, âcre et aigre indiquent, outre la notion de désagrément, d'où provient cette impression désagréable : du caractère irritant de l'émanation pour âcre ${ }^{12}$ et d'une acidité désagréable pour aigre ${ }^{13}$, et ce en vertu d'analogies sensorielles, gustatives en l'occurrence. En outre, une odeur âcre renvoie au toucher (qui picote, qui a des aspérités) et provoque un effet à la gorge.

La multi-sensorialité de la perception des odeurs peut être expliquée par le lieu et le mode d'appréhension communs à certaines sensations ${ }^{14}$. Odorat et goût sont ainsi mis en parallèle du fait de la proximité des lieux où se produit la perception des odeurs et du goût. Lorsque des aliments sont ingérés, les arômes qu'ils dégagent suivent une trajectoire passant en arrière du palais pour atteindre les fosses nasales. Dans cette optique, on peut donc considérer comme tout à fait « normal » d'utiliser des adjectifs dédiés au goût pour décrire les odeurs, puisque le goût apparaît d'emblée comme une perception multi-sensorielle englobant l'odorat.

Le type de sensation plaide également pour un rapprochement entre le goût et odorat. Ceux-ci sont en effet « souvent classés comme <sens chimiques> parce qu'ils sont stimulés par des molécules, alors que la vue, l'ouie et le toucher

12. « Au point de brûler, de prendre à la gorge ». $(P R E)$

13. Notons également que, si les sujets ne s'accordent pas toujours quant à la qualification des odeurs, c'est peut-être aussi dû au fait que les définitions associées aux adjectifs « sensoriels » sont loin d'être uniformes, comme en témoignent les définitions de aigre dans le $P R E$ et le $T L F i$ qui sont respectivement « qui est d'une acidité désagréable au goût ou à l'odorat » et « qui produit une impression piquante, désagréable sur les organes des sens ».

14. Plusieurs explications ont déjà été avancées sur l'existence de combinaisons multi-sensorielles. Parmi elles, on compte tout d'abord la synesthésie qui constitue la perception et l'expression d'une fusion de sensations, alliant aspects sensoriels et linguistiques. L'hypallage est également invoquée en ce qu'elle a « la particularité [...] de proposer plusieurs parcours interprétatifs simultanés » (Legallois, 2004 : 498). Voir également Morrot et al. (2001) et Ramachandran (2006). 
répondent à des stimulations physiques » (cf. Dupire (1987) ; Bessirier \& Guinard (1996 : 227) ; Roudnitska (1987 : 82-83) ; Candau (2000 : 35), entre autres). L'analogie du type de sensation apporte un argument supplémentaire permettant d'expliquer la prépondérance de l'aspect gustatif dans la description des odeurs. Que les odeurs aient enfin des propriétés tactiles n'est pas indissociable de leur vecteur, à savoir l'air, qui peut par exemple être âcre et provoquer la sensation correspondante dans le nez ou dans la gorge de la personne qui les sent.

Dans notre corpus, les propriétés que l'on peut attribuer aux odeurs arrivent, en termes d'occurrences, en première position chez les hédoniques postposés et après les hédoniques purs chez les antéposés ${ }^{15}$. Toutefois, les adjectifs de propriété restent beaucoup plus largement employés en postposition, ce qui est à nouveau à mettre en lien avec le traitement analytique accordé aux bonnes ou aux mauvaises odeurs dont on précise un aspect particulier. Enfin, du point de vue de la diversité lexicale, les propriétés occupent un tiers des hédoniques.

\subsection{Les traits hédonique et intensité}

Le trait « intensité », malgré le peu de moyens linguistiques qui lui sont dédiés que ce soit des adjectifs uniquement hédoniques et intensifs (forte, passée et violente) ou d'autres incluant en plus les traits "propriété » (fade, douceâtre, doucereuse, flétrie et implacable) ou " effet » (affadie et affadissante) - représente plus du tiers des occurrences d'adjectifs hédoniques négatifs ${ }^{16}$. En antéposition, c'est l'adjectif forte qui, comme bonne, est emblématique de la catégorie. Il l'est également parmi les postposés, où il est suivi de près par fade. Nous avons choisi d'inclure dans les hédoniques les adjectifs d'intensité forte et fade pour différentes raisons. Tout d'abord, il est souvent admis par les sujets parlants qu'une odeur forte (pas forcément désagréable) dérange ou monte à la tête et qu'une odeur fade $^{17}$ est une odeur sans caractère (comme l'est un goût fade) et qui déplaît. Les définitions lexicographiques de ces deux adjectifs, ainsi que nos exemples, associent souvent dégoût et intensité :

\subsubsection{Définition lexicographique (TLFi)}

\section{Fade}

[En parlant d'une réalité perceptible par tel ou tel sens] Qui manque de saveur ou de caractère et suscite souvent le dégoût ou un jugement défavorable.

a) Domaine du goût. Qui manque de saveur ou dont la saveur trop peu relevée déplaît au goût. Mets, viande, sauce fade ; une douceur fade (Ac. 1835-1932).

b) Domaine de l'odorat. (Quasi-)anton. suave. L'odeur fade et vaporeuse de sa chevelure me faisait souvent répugnance (MILOSZ, Amour. initiation, 1910, p. 176).

15. Les adjectifs de propriété arrivent en troisième position chez les hédoniques négatifs antéposés après les hédoniques purs et l'intensité. Nous y reviendrons.

16. Signalons l'absence d'adjectifs hédoniques positifs intensifs.

17. De même, une odeur doucereuse est une odeur qui est d'une douceur fade et désagréable. 
c) Domaine de la vue. Désagréable à la vue par son manque d'éclat. Couleur fade (Ac. 1798-1932). (Quasi-)synon. blême, délavé, pâle, terne. À droite, un rideau de palmiers (...) troncs tout gris, sommets inclinés vers le sud, vert fade (FROMENTIN, Voy. Égypte, 1869 , p. 52).

d) Domaine de l'oü̈e. Désagréable à l'oreille par sa monotonie.

Fort

Qui manque de finesse, de délicatesse. Synon. grossier, épais. Les traits gros et forts, osseux, cabossés et comme ébauchés dans de la glaise (GONCOURT, Journal, 1864, p. 91).

\subsubsection{Exemples de Frantext}

- odeur fade de cave moisie ; odeur fade d'enfant malade qui me répugnait ; je m’éloignais de lui, écœurée par l'odeur fade qui sortait de son corps ; à cette fade odeur qui vous met malgré vous le trouble au cœur ;

- forte odeur de nécrose fétide ; forte odeur de pourriture ; cette forte odeur, pas désagréable ; on étouffait dans l'odeur forte qui montait.

Nous pouvons constater que le dictionnaire ne précise pas pour l'odorat, contrairement aux autres sens concernés, en quoi fade est désagréable. Fade indique une neutralisation qualitative de l'odeur qui conduit à la nonreconnaissance du prototype de l'odeur en question. Ainsi, une odeur de rose qui serait fade ne répondrait pas à toutes les qualités normalement requises pour être considérée comme une véritable odeur de rose. C'est alors à nouveau l'aspect gustatif qui apparaît comme saillant dans la transposition multi-sensorielle des odeurs.

\subsection{Les traits hédonique et effet}

Les adjectifs dénotant un effet sont pour la plupart des déverbaux qui impliquent le sens du verbe et dont la sémantique de l'affixe utilisé (-ant, -if, -ible) conforte l'interprétation par l'effet. Cette catégorie a bien été signalée dans les études antérieures et elle est également bien présente dans notre corpus. Les paraphrases suivantes indiquent d'ailleurs qu'une odeur provoque un effet qui peut recevoir à son tour une interprétation hédonique :

- une insoutenable odeur est une odeur que l'on ne peut pas supporter ;

- une odeur agressive est une odeur qui agresse ;

- une caressante odeur est une odeur qui caresse, etc. 
Mais, ce qui ne ressortait pas des travaux précédents ${ }^{18} c^{\prime}$ est que, parmi ces adjectifs d'effet, certains renseignent sur la cause de l'effet et donc, indirectement, sur une propriété de l'odeur (surtout quand l'adjectif est porteur d'une triple interprétation effet, propriété et hédonisme). D'autres, en revanche, ne révèlent rien sur l'odeur elle-même si ce n'est ce que l'expérienceur en pense subjectivement. Ces derniers ne soulignent pas, en somme, en quoi on a affaire à une odeur $X$ ou Y. Ces remarques nous conduisent à distinguer deux grandes sous-catégories d'adjectifs effet-hédoniques qu'ils soient antéposés ou postposés :

- les adjectifs sous-déterminés indiquant, dans l'ensemble, le rejet : écourante, insupportable, intolérable, effroyable, repoussante, agressive, nauséabonde ${ }^{19}$, etc. ;

- les adjectifs multi-sensoriels renseignant directement sur quelques propriétés olfactives : âcre, râpeuse, caressante, fraîche, fétide, etc.

Notre corpus confirme ce que l'opposition entre axes positif et négatif laissait supposer : il y a une multiplicité d'effets pour les hédoniques négatifs, alors qu'il y en a beaucoup moins chez les hédoniques positifs. En termes de diversité lexicale, le positif ne se réalise, en antéposition, que par deux adjectifs effets (caressante et charmante) et par cinq en postposition (attendrissante, enchanteresse, épatante, époustouflante et appétissante). La catégorie effet se place, en revanche, en première position parmi les hédoniques négatifs antéposés et en deuxième position (derrière la catégorie propriétés et devant les hédoniques purs) parmi les postposés. Ces faits rejoignent les observations déjà faites (§3) quant au besoin, pour les sujets parlants, de préciser en quoi une odeur est mauvaise. La prise en compte du nombre d'occurrences des adjectifs hédoniques négatifs dénotant un effet dans notre corpus indique cependant que, même si ce trait présente une grande diversité lexicale, il n'est pas pour autant prépondérant, puisque les catégories propriété et intensité sont, comme on l'a vu supra, les figures majeures en lien avec l'hédonisme.

En somme, le trait effet est un moyen d'indiquer une propriété sans nommer la propriété elle-même. Autrement dit, les adjectifs se rapportant à l'axe effet dénotent des propriétés spécifiques de l'odeur. Et, même si la perception est subjective, essentiellement en raison de la non-tangibilité de l'odeur, l'effet, lui, est un résultat concret en ce sens qu'il est la trace perceptive laissée par une

18. L'odeur elle-même serait conçue comme un effet, c'est-à-dire comme une manifestation indissociable de la personne qui la perçoit : «pour catégoriser des odeurs, les locuteurs [utilisent] des expressions telles que odeur agréable, odeur désagréable, odeur piquante, odeur gênante, odeur réconfortante, etc. [...]. On signifie avec ces expressions [i.e. adj en -able ou en -ant] qu'une odeur (nous) réconforte, (me) gêne, pique (quelqu'un), etc. » (David \& Dubois, en ligne b). Cf. aussi, David et al. (1997) ; Dubois \& Rouby (1997: 17); Dubois (2006 : 104) ; Lenclud (2006 : 10) ; Dubois (2008); Dubois \& Resche-Rigon (2009 : 409). Cette position nous semble un peu forte car, même s'il est indéniable que l'odeur en tant que sensation est subjective, on la conçoit quand même comme un objet odorant en tant que tel, ayant une existence en dehors de la personne qui peut sentir l'odeur et en dehors de la source dont elle émane.

19. Cet adjectif, même s'il indique que la mauvaise odeur provoque un malaise physique allant jusqu'à la nausée, tend cependant à devenir très lexicalisé au point de ne plus percevoir l'effet en premier. On a affaire, en quelque sorte, à un adjectif passe-partout, proche de mauvais, d'où notre choix de l'inclure dans la première sous-classe. 
certaine propriété de l'odeur. Il n'est donc pas certain que les odeurs soient pour les locuteurs tout simplement des effets (cf. Kleiber \& Vuillaume 2011). Il ressort globalement qu'avec odeur, l'analyse se fait plus facilement par le biais des effets (vs. pour la vue ou le goût pour lesquels nous possédons quelques stabilisateurs lexicaux tels que rouge, noir, salé, sucré, etc.).

\section{CONCLUSION}

Notre étude consacrée à la dimension hédonique des odeurs peut paraître limitée et est sûrement loin d'être terminée. Elle a toutefois montré que les odeurs ne sont pas simplement des effets et que l'on peut les saisir via leurs propriétés. Et, même si on ne peut pas aller jusqu'à soutenir qu'il existe des types d'odeurs, nos résultats font néanmoins émerger une certaine stabilité dans le domaine olfactif à travers la prégnance des axes air, toucher et goût. Les deux premiers sont fortement liés et semblent dessiner le prototype d'une bonne odeur qui serait, d'après notre corpus, une odeur chaleureuse et caressante. Autrement dit, une bonne odeur (même si ce n'est qu'une appréciation) doit avoir des propriétés pour plaire, lesquelles propriétés se manifestent surtout à travers les effets qu'elles provoquent. D'autres adjectifs ${ }^{20}$ - tels que chaude, fraîche, tiède, pénétrante, volatile - confortent notre analyse en termes d'air et de toucher. Du côté négatif, c'est l'adjectif âcre, porteur lui aussi des traits liés au domaine tactile, qui constituerait le représentant type d'une mauvaise odeur. En ce qui concerne les adjectifs relevant de sensations autres qu'olfactives, nous n'avons fait que confirmer linguistiquement ce que les sciences dures ont avancé pour leur domaine. Par ailleurs, la prise en compte d'appétissante faisant partie des rares adjectifs possédant simultanément les trois traits hédonique, effet et propriété gustative va dans le même sens.

Nos résultats confirment également l'analyse de G. Kleiber et $\mathrm{M}$. Vuillaume (2011) et rejoignent les données de P. Blumenthal (2011) qui a répertorié, parmi les lexèmes les plus spécifiques au mot odeur, ceux de saveur, chaleur et goût. Dans le même ordre d'idées, la prégnance des adjectifs hédoniques purs en antéposition ainsi que les collocations bonne/mauvaise/forte odeur vont de pair avec une perception de type holistique, tandis que le processus analytique est plutôt activé à l'aide d'adjectifs postposés comportant plusieurs traits, lorsque l'on décrit de mauvaises odeurs. Dans tous les cas, l'adjectif contribue à la construction de catégories cognitives (cf. Cance et al. 2009).

L'examen d'un corpus comme Frantext aura enfin montré qu'il n'y a quasiment pas de combinaisons impossibles avec odeur. Des séquences comme une odeur verte sont, en effet, tout à fait acceptables par le biais de l'hypallage. Mais tous les adjectifs ne sont pas égaux quant aux informations qu'ils apportent sur

20. Une rapide analyse de l'entourage verbal des odeurs semble aller dans le même sens étant donné le nombre élevé de verbes comme pénétrer, s'exhaler, s'évaporer, se diffuser, émaner, etc. 
les odeurs, certains étant spécialisés dans l'olfaction - comme odoriférant ${ }^{21}-$, d'autres véhiculant plusieurs traits sémantiques explicitant les propriétés des odeurs. Cette diversité peut également être marquée syntaxiquement par la coordination d'adjectifs qui associent alors des propriétés auparavant isolées. Ces associations, de même que les autres axes qui structurent les odeurs - tels que source, identification, temporalité et ceux qui ont été abordés ici - mériteraient un examen approfondi.

\section{Références}

[FRANTEXT] http://www.frantext.fr/

[PRE] Le Petit Robert Électronique, Cédérom, Dictionnaire Le Robert.

[TLFI] http://atilf.atilf.fr/

ABEILlÉ A. \& GodARD D. (1999), "La position de l'adjectif épithète en français : le poids des mots ", Recherches Linguistiques de Vincennes 28, 9-32.

BARTNING I. (1980), Remarques sur la syntaxe et la sémantique des pseudo-adjectifs dénominaux en français, Stockholm : Almqvist \& Wiksell International.

BESSIRIER J.-M. \& GUINARD J.-X. (1996), "Attention, ça pique... ! Le troisième sens chimique ", Psychologie française 41 (3), 227-235.

BLINKENBERG A. (1928), L'ordre des mots en français moderne, vol. 1, Copenhague : Det kgl. Danske videnskabernes selskab.

Blumenthal P. (2011), "Odeur - évolution des profils combinatoires ", Langages 181, Paris : Armand Colin (ce volume).

Boısson C. (1997), "La dénomination des odeurs : variations et régularités linguistiques ", Intellectica 24, 29-49.

Buck C.D. (1949), A Dictionary of Selected Synonyms in the Principal Indo-European Languages. A Contribution to the History of Ideas, Chicago: Chicago University Press.

CANCE C. et al. (2009), "Des adjectifs construits pour qualifier le monde ", in D. Dubois (éd.), Le sentir et le dire. Concepts et méthodes en psychologie et linguistique cognitives, Paris : L'Harmattan, 279-308.

CANdAu J. (2000), Mémoire et expériences olfactives. Anthropologie d'un savoir-faire sensoriel, Paris : Presses Universitaires de France.

Candau J. \& Jeanjean A. (2006), "Des odeurs à ne pas regarder ", Terrain 47, 51-68.

ChAuveix S. \& EGOROFF C. (2003), "Mise au point d'une liste de descripteurs sensoriels : une nouvelle approche basée sur la catégorisation ", Psychologie française 48 (4), 79-89.

Chollet S., VAlentin D. \& ABDI H. (2003), "Les mots du vin : experts et novices diffèrent-ils quand ils décrivent des vins ? ", Corpus 2, 183-199.

Chréa C. \& Valentin D. (2007), "Les odeurs : une question culturelle ", Cerveau \& Psycho 21 , 40-44.

CHRÉA C. et al. (2005), "Semantics, typicality and odor representation: a cross-cultural study", Chemical Senses 30, 37-49.

21. Il serait d'ailleurs intéressant de relever les adjectifs spécifiquement liés à l'olfaction. 
CORBIN A. (1982), Le miasme et la jonquille. L'odorat et l'imaginaire social auX XVIII ${ }^{e}-X I X^{e}$ siècles, Paris : Aubier Montaigne.

DAVID S. (2002), “Linguistic Expressions for Odors in French”, in C. Rouby et al. (eds), Olfaction, Taste, and Cognition, Cambridge: Cambridge University Press, 82-99.

DAvid S. \& DuBoIS D. (en ligne a), " Langage et représentations de l'odeur. Des catégories d'odeurs ". (http://www.culture.gouv.fr/culture/dglf/francais-aime/parfums/lcpe/categorieodeur.htm)

DAVID S. \& DuBoIS D. (en ligne b), "Langage et représentations de l'odeur. Quelques propriétés linguistiques des noms renvoyant au domaine olfactif ". (http://www.culture.gouv.fr/culture/ dglf/francais-aime/parfums/lcpe/proprietes-linguistiques.htm)

DAVID S. \& Rouby C. (2002), "Groupements d'odeurs ou catégories ? Questions de méthodologie ", Cahiers du LCPE 6, 29-68.

DAVID S. et al. (1997), "L'expression des odeurs en français : analyse lexicale et représentation cognitive ", Intellectica 24, 51-83.

Dubols D. (2000), "Categories as Acts of Meaning: The Case of Categories in Olfaction and Audition", Cognitive Science Quarterly 1, 35-68.

DuBols D. (2006), " Des catégories d'odorants à la sémantique des odeurs. Une approche cognitive de l'olfaction ", Terrain 47, 89-106.

DuBoıs D. (2008), "Sens communs et sens commun : expériences sensibles, connaissance(s) ou doxa ? ", Langages 170, 41-53.

DuBoIs D. \& RESCHE-RIGON P. (2009), "Langue, discours et cognition : une approche sémiophysique de la construction du sens linguistique ", in $\mathrm{E}$. Havu et al. (éds), La langue en contexte, Helsinki : Société Néophilologique, 405-418.

Dubois D. \& Rouby C. (1997), "Une approche de l'olfaction : du linguistique au neuronal ", Intellectica 24, 9-20.

DUPIRE M. (1987), " Des goûts et des odeurs : classification et universaux ", L'Homme 104, 5-25.

FORSGREN M. (1978), La place de l'adjectif épithète en français contemporain. Étude quantitative et sémantique, Uppsala : Acta Universitatis Upsaliensis.

GoEs J. (1993), "À la recherche d'une définition de l'adjectif ", L'information grammaticale 58, 11-14.

GOES J. (1999), L'adjectif. Entre nom et verbe, Louvain-la-Neuve : Duculot.

Holley A. (1997), "Le physiologiste et la catégorisation des odeurs ", Intellectica 24, 21-27.

Hombert J.-M. (1992), "Terminologie des odeurs dans quelques langues du Gabon ", Pholia 7 , 61-65.

HONESTE M.-L. (2005), "Approche cognitive de la fonction adjectivale ", in J. François (éd.), L'Adjectif en français et à travers les langues, Caen : Presses Universitaires de Caen, 135-149.

Kleiber G. \& VUillaume M. (2011), "Sémantique des odeurs ", Langages 181, Paris : Armand Colin (ce volume).

LARSSON B. (1994), La place et le sens des adjectifs épithètes de valorisation positive, Lund : Lund University Press.

LE BIDOIS G. \& LE BIDoIS R. (1967), Syntaxe du français moderne : ses fondements historiques et psychologiques, Paris : Picard. 
LEGalloIs D. (2004), "Synesthésie adjectivale, sémantique et psychologie de la forme : la transposition au cœur du lexique ", in J. François (éd.), L'adjectif en français et à travers les langues, Caen : Presses Universitaires de Caen, 493-506.

LENCLUD G. (2006), " La nature des odeurs (remarques) ", Terrain 47, 5-18.

Morrot G., Brochet F. \& Dubourdieu D. (2001), “The Color of Odors”, Brain and Language 79 (2), 309-320.

NoAiLly M. (1999), L'adjectif en français, Paris : Ophrys.

NøLKE H. (2000), "L'ordre des mots : plusieurs adjectifs épithètes antéposés ", in A. Englebert et al. (éds), De la grammaire des formes à la grammaire du sens, Niemeyer : Tübingen, 519-526.

RamachandRan V. (2006), "La synesthésie ou la confusion des sens ", Cerveau et Psycho 14, 772-77.

RICAUD P. (2004), "Le jugement olfactif et ses implications sociales ", Voir 28/29, 34-41.

Rouby C. \& Sicard G. (1997), " Des catégories d'odeurs ? ", in D. Dubois (éd.), Catégorisation et cognition : de la perception au discours, Paris : Kimé, 59-81.

Roudnitska E. (1987), "L'univers du parfum ", Autrement 92, 80-87.

SCHLEIDT M., Neumann P. \& MorishitA H. (1988), "Pleasure and disgust, memories and associations of pleasant and unpleasant odors in Germany and Japan", Chemical Senses 13 (2), 279-283.

StATI S. (1979), La sémantique des adjectifs. Essai d'analyse componentielle appliquée aux langues romanes, Paris : Fayard.

WierzBicka A. (1988), The Semantics of Grammar, Amsterdam/Philadelphia: John Benjamins.

WILMET M. (1980), "Antéposition et postposition de l'épithète qualificative en français contemporain ", Travaux de linguistique 7, 179-201.

WILMET M. (1981), "La place de l'épithète qualificative en français contemporain : étude grammaticale et stylistique ", Revue de linguistique romane 45, 17-73. 